\title{
Retrospective Cost Model Refinement for On-line Estimation of Constant and Time-Varying Flight Parameters
}

\author{
Jiapeng Zhong , Jin Yan † Yousaf Rahman ‡ Changhong Wang §, \\ and Dennis S. Bernstein \\ University of Michigan, 1320 Beal Ave., Ann Arbor, MI 48109
}

\begin{abstract}
In this paper we apply retrospective cost model refinement (RCMR) to parameter estimation. To gain insight into the accuracy and speed of convergence of the RCMR estimates, we consider aircraft dynamics with uncertain entries in the dynamics and input matrices. We consider scenarios that include multiple uncertain parameters, alternative measurements, and sensor noise. All of the examples are discrete time, as required by RCMR. To account for the structure of the uncertain parameters, we develop a Jacobianbased technique for constructing the appropriate feedback structure. We use this approach to account for the appearance of stability derivatives in the dynamics matrix, and we use this technique to estimate airspeed variations in the vicinity of trimmed flight.
\end{abstract}

\section{Introduction}

System identification is concerned with using input-output data to construct empirical models. In many cases, some components of the system are well-modeled, and the goal is to use input-output data to improve estimates of poorly modeled components. These components may be connected in cascade, parallel, or feedback with the well-modeled components, and they may be static or dynamic. This problem is known as model updating, model correction, model calibration, and model refinement [1-6].

The most common model-refinement problem is parameter estimation, where data are used to improve estimates of parameters in a model whose structure is known. Parameter estimation is related to, but distinct from, state estimation, where the states evolve due to external inputs and their interaction with other states. In contrast, an unknown parameter may either be constant or time-varying in a pre-specified manner.

The close relationship between parameter estimation and state estimation is evident from the widespread use of state-estimation techniques for parameter estimation. In particular, the extended Kalman filter can be used with a linearized model to propagate state and parameter estimates [7]. Alternatively, techniques developed for nonlinear state estimation can be applied to parameter estimation [8-11].

In the present paper we revisit the retrospective cost approach to model refinement (RCMR) [12-15]. RCMR can be used to estimate the dynamics of a possibly dynamic subsystem in feedback interconnection

\footnotetext{
*Graduate Student, Control Science and Engineering Department, Harbin Institute of Technology, Harbin, China

$\dagger$ Graduate Student, Aerospace Engineering Department, University of Michigan, Ann Arbor

${ }^{\ddagger}$ Graduate Student, Aerospace Engineering Department, University of Michigan, Ann Arbor

§Professor, Control Science and Engineering Department, Harbin Institute of Technology, Harbin, China

`Professor, Aerospace Engineering Department, University of Michigan, Ann Arbor
} 
with a main subsystem; the unknown subsystem is assumed to be inaccessible in the sense that its inputs and outputs are not measured. A special case of an inaccessible subsystem occurs when the unknown subsystem is static; in this case, inaccessible subsystem identification is equivalent to parameter estimation.

The goal of the present paper is to apply RCMR to parameter estimation. To gain insight into the accuracy and speed of convergence of the RCMR estimates, we consider aircraft dynamics with uncertain entries in the dynamics and input matrices. These scenarios include multiple uncertain parameters, alternative measurements, and sensor noise. All of the examples are discrete time, as required by RCMR. To account for the structure of the uncertain parameters, we develop a Jacobian-based technique for constructing the appropriate feedback structure. We use this approach to account for the appearance of stability derivatives in the dynamics matrix, and we use this technique to estimate variations of the airspeed in the vicinity of trimmed flight.

\section{Problem Statement}

Consider the MIMO discrete-time main system

$$
\begin{aligned}
x(k+1) & =A x(k)+B u(k)+D_{1} w(k), \\
y(k) & =C x(k), \\
y_{0}(k) & =E_{1} x(k)+v(k),
\end{aligned}
$$

where $x(k) \in \mathbb{R}^{n}, y(k) \in \mathbb{R}^{l_{y}}, y_{0}(k) \in \mathbb{R}^{l_{y_{0}}}, u(k) \in \mathbb{R}^{l_{u}}, w(k) \in \mathbb{R}^{l_{w}}$, and $k \geq 0$. The main system (1)-(3) is interconnected with the unknown subsystem modeled by

$$
u(k)=G_{\mathrm{s}}(q) y(k),
$$

where $q$ is the forward shift operator. The system (1)-(4) represents the true system. We assume that the excitation signal $w(k)$ is known. $v(k)$ denotes measurement noise.

Next, we assume a model of the main system of the form

$$
\begin{aligned}
\hat{x}(k+1) & =\hat{A} \hat{x}(k)+\hat{B} \hat{u}(k)+\hat{D}_{1} w(k), \\
\hat{y}(k) & =\hat{C} \hat{x}(k), \\
\hat{y}_{0}(k) & =\hat{E}_{1} \hat{x}(k),
\end{aligned}
$$

where $\hat{x}(k) \in \mathbb{R}^{\hat{n}}, \hat{y}(k) \in \mathbb{R}^{l_{\hat{y}}}, \hat{y}_{0}(k) \in \mathbb{R}^{l_{y_{0}}}, \hat{u}(k) \in \mathbb{R}^{l_{\hat{u}}}$. The model of the main system is interconnected with the subsystem model

$$
\hat{u}(k)=\hat{G}_{\mathrm{s}}(q) \hat{y}(k) .
$$

The goal is to estimate a subsystem model $\hat{G}_{\mathrm{S}}(q)$ by minimizing a cost function based on the performance variable

$$
z(k) \triangleq \hat{y}_{0}(k)-y_{0}(k) \in \mathbb{R}^{l_{z}}
$$

We estimate $\hat{G}_{\mathrm{S}}(q)$ by retrospectively reconstructing the signal $\hat{u}(k)$ that minimizes the performance at the current time step. The reconstruction of $\hat{u}(k)$ uses minimal modeling information about the true system (1)-(3), namely, a limited number of Markov parameters. We then use $\hat{u}(k)$ and $\hat{y}(k)$ to construct $\hat{G}_{\mathrm{s}}(q)$. Figure 1 illustrates the model-refinement architecture.

\section{Retrospective Control Model Refinement}

We begin by defining Markov parameters of the main system model $\hat{G}(q)$. For $i \geq 1$, let

$$
H_{i} \triangleq \hat{E}_{1} \hat{A}^{i-1} \hat{B}
$$




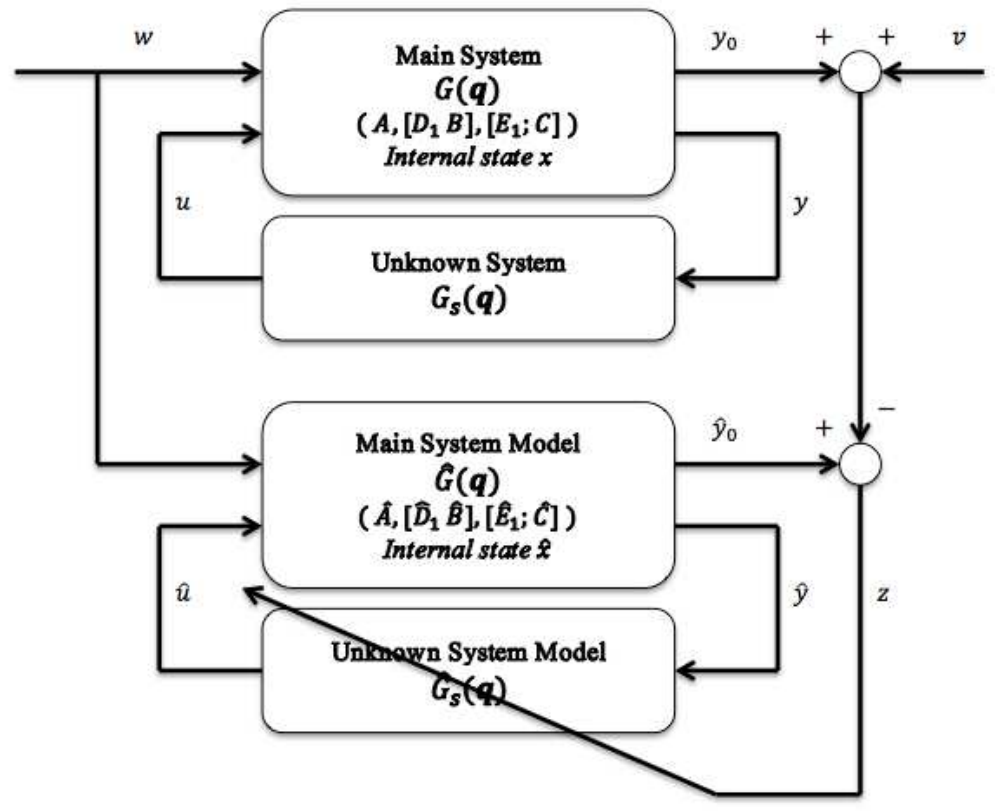

Figure 1. Model-refinement architecture.

Therefore, $H_{1}=\hat{E}_{1} \hat{B}$ and $H_{2}=\hat{E}_{1} \hat{A} \hat{B}$. Let $r$ be a positive integer. Then, for all $k \geq r$,

$$
\hat{x}(k)=\hat{A}^{r} \hat{x}(k-r)+\sum_{i=1}^{r} \hat{A}^{i-1} \hat{B} \hat{u}(k-i)+\sum_{i=1}^{r} \hat{A}^{i-1} \hat{D}_{1} w(k-i),
$$

and thus

$$
z(k)=\hat{E}_{1} \hat{A}^{r} \hat{x}(k-r)+\sum_{i=1}^{r} \hat{E}_{1} \hat{A}^{i-1} \hat{D}_{1} w(k-i)-y_{0}(k)+\overline{\mathcal{H}} \bar{U}(k-1),
$$

where $\overline{\mathcal{H}} \triangleq\left[\begin{array}{lll}H_{1} & \cdots & H_{r}\end{array}\right] \in \mathbb{R}^{l_{z} \times r l_{\hat{u}}}$, and $\bar{U}(k-1) \triangleq\left[\begin{array}{lll}\hat{u}^{\mathrm{T}}(k-1) & \cdots & \hat{u}^{\mathrm{T}}(k-r)\end{array}\right]^{\mathrm{T}}$.

Next, we rearrange the columns of $\overline{\mathcal{H}}$ and the components of $\bar{U}(k-1)$ and partition the resulting matrix and vector so that

$$
\overline{\mathcal{H}} \bar{U}(k-1)=\mathcal{H}^{\prime} U^{\prime}(k-1)+\mathcal{H} U(k-1),
$$

where $\mathcal{H}^{\prime} \in \mathbb{R}^{l_{z} \times\left(r l_{\hat{u}}-l_{U}\right)}, \mathcal{H} \in \mathbb{R}^{l_{z} \times l_{U}}, U^{\prime}(k-1) \in \mathbb{R}^{r l_{\hat{u}}-l_{U}}$, and $U(k-1) \in \mathbb{R}^{l_{U}}$. Then, we can rewrite (12) as

$$
z(k)=\mathcal{S}(k)+\mathcal{H} U(k-1),
$$

where

$$
\mathcal{S}(k) \triangleq \hat{E}_{1} \hat{A}^{r} \hat{x}(k-r)+\sum_{i=1}^{r} \hat{E}_{1} \hat{A}^{i-1} \hat{D}_{1} w(k-i)-y_{0}(k)+\mathcal{H}^{\prime} U^{\prime}(k-1) .
$$

For example, $\overline{\mathcal{H}}=\left[\begin{array}{lll}H_{1} & H_{2} & H_{3}\end{array}\right]$,

$$
\mathcal{H}^{\prime}=\left[\begin{array}{ll}
H_{1} & H_{2}
\end{array}\right], \quad U^{\prime}(k-1)=\left[\begin{array}{c}
\hat{u}(k-1) \\
\hat{u}(k-2)
\end{array}\right],
$$


and $\mathcal{H}=H_{3}, \quad U(k-1)=\hat{u}(k-3)$. Next, we rewrite (14) with a delay of $k_{j}$ time steps, where $0 \leq k_{1} \leq$ $k_{2} \leq \cdots \leq k_{s}$, in the form

$$
z\left(k-k_{j}\right)=\mathcal{S}_{j}\left(k-k_{j}\right)+\mathcal{H}_{j} U_{j}\left(k-k_{j}-1\right)
$$

where (15) becomes

$$
\mathcal{S}_{j}\left(k-k_{j}\right) \triangleq \hat{E}_{1} \hat{A}^{r} \hat{x}\left(k-k_{j}-r\right)+\sum_{i=1}^{r} \hat{E}_{1} \hat{A}^{i-1} \hat{D}_{1} w\left(k-k_{j}-i\right)-y_{0}\left(k-k_{j}\right)+\mathcal{H}_{j}^{\prime} U_{j}^{\prime}\left(k-k_{j}-1\right)
$$

and (13) becomes

$$
\overline{\mathcal{H}} \bar{U}\left(k-k_{j}-1\right)=\mathcal{H}_{j}^{\prime} U_{j}^{\prime}\left(k-k_{j}-1\right)+\mathcal{H}_{j} U_{j}\left(k-k_{j}-1\right),
$$

where $\mathcal{H}_{j}^{\prime} \in \mathbb{R}^{l_{z} \times\left(r l_{\hat{u}}-l_{U_{j}}\right)}, \mathcal{H}_{j} \in \mathbb{R}^{l_{z} \times l_{U_{j}}}, U_{j}^{\prime}\left(k-k_{j}-1\right) \in \mathbb{R}^{r l_{\hat{u}}-l_{U_{j}}}$, and $U_{j}\left(k-k_{j}-1\right) \in \mathbb{R}^{l_{U_{j}}}$. Now, by stacking $z\left(k-k_{1}\right), \ldots, z\left(k-k_{s}\right)$, we define the extended performance

$$
Z(k) \triangleq\left[\begin{array}{lll}
z^{\mathrm{T}}\left(k-k_{1}\right) & \cdots & z^{\mathrm{T}}\left(k-k_{s}\right)
\end{array}\right]^{\mathrm{T}} \in \mathbb{R}^{s l_{z}} .
$$

Therefore,

$$
Z(k) \triangleq \tilde{\mathcal{S}}(k)+\tilde{\mathcal{H}} \tilde{U}(k-1)
$$

where $\tilde{\mathcal{S}}(k) \triangleq\left[\mathcal{S}^{\mathrm{T}}\left(k-k_{1}\right) \cdots \mathcal{S}^{\mathrm{T}}\left(k-k_{s}\right)\right]^{\mathrm{T}} \in \mathbb{R}^{s l_{z}}, \tilde{\mathcal{H}} \in \mathbb{R}^{s l_{z} \times l_{\tilde{U}}}$, and $\tilde{U}(k-1) \in \mathbb{R}^{l_{\tilde{U}}}$. The vector $\tilde{U}(k-1)$ is formed by stacking $U_{1}\left(k-k_{1}-1\right), \ldots, U_{s}\left(k-k_{s}-1\right)$ and removing repetitions of components. For example, with $k_{1}=0$ and $k_{2}=1$, stacking $U_{1}(k-1)=\left[\begin{array}{c}\hat{u}(k-1) \\ \hat{u}(k-2)\end{array}\right]$ and $U_{2}(k-2)=\hat{u}(k-2)$ results in $\tilde{U}(k-1)=\left[\begin{array}{c}\hat{u}(k-1) \\ \hat{u}(k-2)\end{array}\right]$. The coefficient matrix $\tilde{\mathcal{H}}$ consists of the entries of $\mathcal{H}_{1}, \ldots, \mathcal{H}_{s}$ arranged according to the structure of $\tilde{U}(k-1)$. Furthermore, we assume that the last entry of $\tilde{U}(k-1)$ is a component of $\hat{u}(k-r)$.

Next, we define the retrospective performance

$$
\hat{z}\left(k-k_{j}\right) \triangleq \mathcal{S}_{j}\left(k-k_{j}\right)+\mathcal{H}_{j} U_{j}^{*}\left(k-k_{j}-1\right),
$$

where the actual past subsystem outputs $U_{j}\left(k-k_{j}-1\right)$ in (16) are replaced by the surrogate subsystem outputs $U_{j}^{*}\left(k-k_{j}-1\right)$. The extended retrospective performance for (21), which is defined as

$$
\hat{Z}(k) \triangleq\left[\begin{array}{lll}
\hat{z}^{\mathrm{T}}\left(k-k_{1}\right) & \cdots & \hat{z}^{\mathrm{T}}\left(k-k_{s}\right)
\end{array}\right]^{\mathrm{T}} \in \mathbb{R}^{s l_{z}},
$$

is given by

$$
\hat{Z}(k)=\tilde{\mathcal{S}}(k)+\tilde{\mathcal{H}} \tilde{U}^{*}(k-1),
$$

where the components of $\tilde{U}^{*}(k-1) \in \mathbb{R}^{l} \tilde{U}$ are components of $U_{1}^{*}\left(k-k_{1}-1\right), \ldots, U_{s}^{*}\left(k-k_{s}-1\right)$ ordered in the same way as the components of $\tilde{U}(k-1)$. Subtracting (20) from (23) yields

$$
\hat{Z}(k)=Z(k)-\tilde{\mathcal{H}} \tilde{U}(k-1)+\tilde{\mathcal{H}} \tilde{U}^{*}(k-1) .
$$

Finally, we define the retrospective cost function

$$
J\left(\tilde{U}^{*}(k-1), k\right) \triangleq \hat{Z}^{\mathrm{T}}(k) R(k) \hat{Z}(k),
$$

where $R(k) \in \mathbb{R}^{s l_{z} \times s l_{z}}$ is a positive-definite performance weighting. The goal is to determine refined subsystem outputs $\tilde{U}^{*}(k-1)$ that would have provided better performance than the subsystem outputs $U(k)$ that were applied to the system. The refined subsystem outputs values $\tilde{U}^{*}(k-1)$ are subsequently used to update the subsystem estimate. 


\section{A. Cost Function Optimization with Adaptive Regularization}

To ensure that (25) has a global minimizer, we consider the regularized cost

$$
\bar{J}\left(\tilde{U}^{*}(k-1), k\right) \triangleq \hat{Z}^{\mathrm{T}}(k) R(k) \hat{Z}(k)+\eta(k) \tilde{U}^{* \mathrm{~T}}(k-1) \tilde{U}^{*}(k-1),
$$

where $\eta(k)=\bar{\eta} z^{\mathrm{T}}(k) z(k)$ and $\bar{\eta} \geq 0$. Substituting (24) into (26) yields

$$
\bar{J}\left(\tilde{U}^{*}(k-1), k\right)=\tilde{U}^{*}(k-1)^{\mathrm{T}} \mathcal{A}(k) \tilde{U}^{*}(k-1)+\mathcal{B}(k) \tilde{U}^{*}(k-1)+\mathcal{C}(k),
$$

where

$$
\begin{aligned}
& \mathcal{A}(k) \triangleq \tilde{\mathcal{H}}^{\mathrm{T}} R(k) \tilde{\mathcal{H}}+\eta(k) I_{l_{\tilde{U}}} \\
& \mathcal{B}(k) \triangleq 2 \tilde{\mathcal{H}}^{\mathrm{T}} R(k)[Z(k)-\tilde{\mathcal{H}} \tilde{U}(k-1)], \\
& \mathcal{C}(k) \triangleq Z^{\mathrm{T}}(k) R(k) Z(k)-2 Z^{\mathrm{T}}(k) R(k) \tilde{\mathcal{H}} \tilde{U}(k-1)+\tilde{U}^{\mathrm{T}}(k-1) \tilde{\mathcal{H}}^{\mathrm{T}} R(k) \tilde{\mathcal{H}} \tilde{U}(k-1) .
\end{aligned}
$$

If either $\tilde{\mathcal{H}}$ has full column rank or $\eta(k)>0$, then $\mathcal{A}(k)$ is positive definite. In this case, $\bar{J}\left(\tilde{U}^{*}(k-1), k\right)$ has the unique global minimizer

$$
\tilde{U}^{*}(k-1)=-\frac{1}{2} \mathcal{A}^{-1}(k) \mathcal{B}(k) .
$$

\section{B. Subsystem Modeling}

The subsystem output $\hat{u}(k)$ is given by the exactly proper time-series model of order $n_{\mathrm{c}}$ given by

$$
\hat{u}(k)=\sum_{i=1}^{n_{\mathrm{c}}} M_{i}(k) \hat{u}(k-i)+\sum_{i=0}^{n_{\mathrm{c}}} N_{i}(k) \hat{y}(k-i),
$$

where, for all $i=0,1, \ldots, n_{\mathrm{c}}, M_{i}(k) \in \mathbb{R}^{l_{\hat{u}} \times l_{\hat{u}}}, N_{i}(k) \in \mathbb{R}^{l_{\hat{u}} \times l_{\hat{y}}}$. The subsystem output (32) can be expressed as $\hat{u}(k)=\theta(k) \phi(k)$, where $\theta(k) \in \mathbb{R}^{l_{\hat{u}} \times\left[n_{\mathrm{c}}\left(l_{\hat{u}}+l_{\hat{y}}\right)+l_{\hat{y}}\right]}$ and $\phi(k) \in \mathbb{R}^{n_{\mathrm{c}}\left(l_{\hat{u}}+l_{\hat{y}}\right)+l_{\hat{y}}}$,

$$
\begin{aligned}
& \theta(k) \triangleq\left[\begin{array}{lllll}
M_{1}(k) & \cdots & M_{n_{\mathrm{c}}}(k) N_{0}(k) \cdots N_{n_{\mathrm{c}}}(k)
\end{array}\right],
\end{aligned}
$$

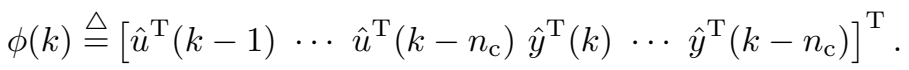

If $n_{\mathrm{c}}=0$, then

$$
\hat{u}(k)=\theta(k) \hat{y}(k)=N_{0}(k) \hat{y}(k) .
$$

\section{Recursive Least Squares Update}

Let $d>0$ such that $\tilde{U}^{*}(k-1)$ contains $u^{*}(k-d)$, and define the retrospective cost function

$$
J_{\mathrm{R}}(\theta(k)) \triangleq \sum_{i=1}^{k} \lambda^{k-i}\left\|u^{* \mathrm{~T}}(k-d)-\phi^{\mathrm{T}}(k-d-1) \theta^{\mathrm{T}}(k)\right\|^{2}+\lambda^{k}(\theta(k)-\theta(0)) P^{-1}(0)(\theta(k)-\theta(0))^{\mathrm{T}},
$$

where $\phi(k-d)$ is given by $(34),\|\cdot\|$ is the Euclidean norm, and $\lambda(k) \in(0,1]$ is the forgetting factor. Minimizing the cumulative cost function yields retrospective cost optimization (RCO)

$$
\begin{aligned}
\theta^{\mathrm{T}}(k) & =\theta^{\mathrm{T}}(k-1)+P(k-1) \phi(k-d-1) \cdot\left[\phi^{\mathrm{T}}(k-d) P(k-1) \phi(k-d-1)+\lambda(k)\right]^{-1} \\
& \cdot\left(u^{*}(k-d)-\phi^{\mathrm{T}}(k-d-1) \theta^{\mathrm{T}}(k-1)\right) .
\end{aligned}
$$


The error covariance is updated by

$$
\begin{aligned}
P(k) & =\lambda^{-1}(k) P(k-1)-\lambda^{-1}(k) P(k-1) \phi(k-d-1) \cdot\left[\phi^{\mathrm{T}}(k-d-1) P(k-1) \phi(k-d)+\lambda(k)\right]^{-1} \\
& \cdot \phi^{\mathrm{T}}(k-d-1) P(k-1) .
\end{aligned}
$$

We initialize the error covariance matrix as $P(0)=\beta I$, where $\beta>0$.

\section{Mass-Spring Example: Stiffness Estimation}

Example IV.1. We consider the mass-spring-damper structure modeled by

$$
m \ddot{q}+c \dot{q}+\kappa q=w,
$$

where $m, c$, and $\kappa$ are the mass, damping, and stiffness, respectively, and $w$ is the force input. We obtain the state-space representation

$$
\begin{aligned}
{\left[\begin{array}{c}
\dot{q} \\
\ddot{q}
\end{array}\right] } & =\left[\begin{array}{cc}
0 & 1 \\
-\frac{\kappa}{m} & -\frac{c}{m}
\end{array}\right]\left[\begin{array}{c}
q \\
\dot{q}
\end{array}\right]+\left[\begin{array}{c}
0 \\
\frac{1}{m}
\end{array}\right] w, \\
y & =\left[\begin{array}{ll}
0 & 1
\end{array}\right]\left[\begin{array}{c}
q \\
\dot{q}
\end{array}\right]+v,
\end{aligned}
$$

where $q$ and $\dot{q}$ are the position and velocity, respectively, of the mass. The parameters are chosen as $m=1$, $c=5$, and $\kappa=10$. The goal is to estimate $\kappa$ with the initial estimate of $\hat{\kappa}(0)=0$.

Using Euler discretizion with sample time $T_{s}$ yields

$$
\begin{aligned}
{\left[\begin{array}{c}
x_{1}(k+1) \\
x_{2}(k+1)
\end{array}\right] } & =\left[\begin{array}{cc}
1 & T_{s} \\
-\frac{\kappa T_{s}}{m} & 1-\frac{c T_{s}}{m}
\end{array}\right]\left[\begin{array}{l}
x_{1}(k) \\
x_{2}(k)
\end{array}\right]+\left[\begin{array}{c}
0 \\
\frac{T_{s}}{m}
\end{array}\right] w(k), \\
y(k) & =\left[\begin{array}{ll}
0 & 1
\end{array}\right]\left[\begin{array}{l}
x_{1}(k) \\
x_{2}(k)
\end{array}\right]+v(k),
\end{aligned}
$$

where, $x_{1}(k) \triangleq q\left(k T_{s}\right)$ and $x_{2}(k) \triangleq \dot{q}\left(k T_{s}\right)$. We define $\hat{A}_{\text {closed-loop }} \triangleq \hat{A}+\hat{B} \hat{\kappa} \hat{C}$, where $\hat{B} \triangleq\left[\begin{array}{c}0 \\ -\frac{T_{s}}{m}\end{array}\right]$ and $\hat{C} \triangleq\left[\begin{array}{ll}1 & 0\end{array}\right]$. The estimate of $\kappa$ is given by $\hat{\kappa}(k)=\theta(k)$. The initial conditions are assumed to be $x_{1}(0)=0.1$ and $x_{2}(0)=0.01$, which are unknown. Let the sensor noise $v$ be Gaussian white noise with mean $\mu_{v}$ and variance $\sigma_{v}^{2}$. Furthermore, $P(0)=0.6, \lambda=0.999, \eta=0, \mu_{v}=0$, and $\tilde{\mathcal{H}}=\left[\begin{array}{ll}H_{1} & H_{2}\end{array}\right]$, which are the first and second Markov parameters of $\hat{G}$. The input is $w(k)=0.8 \sin (\pi k / 20)$. RCMR is turned on at $k=20$ steps. Figure 2 shows the performance $z$ and the estimated $\hat{\kappa}$.

We next consider the effect of sensor noise. We consider the same estimation problem with white sensor noise. We define SNR $\triangleq \frac{\sigma_{\bar{y}_{0}}^{2}}{\sigma_{v}^{2}}$, where $\sigma_{\bar{y}_{0}}^{2}$ is the variance of the output signal $\bar{y}_{0}$. Figure 3 shows the effect of sensor noise on RCMR. As sensor noise is increased, the accuracy of the parameter identification is reduced.

\section{Estimation of a Repeated Parameter}

We next consider the case where one unknown parameter appears in multiple locations within the model. Consider the continuous-time system

$$
\dot{x}=A_{c} x+D_{c} w,
$$



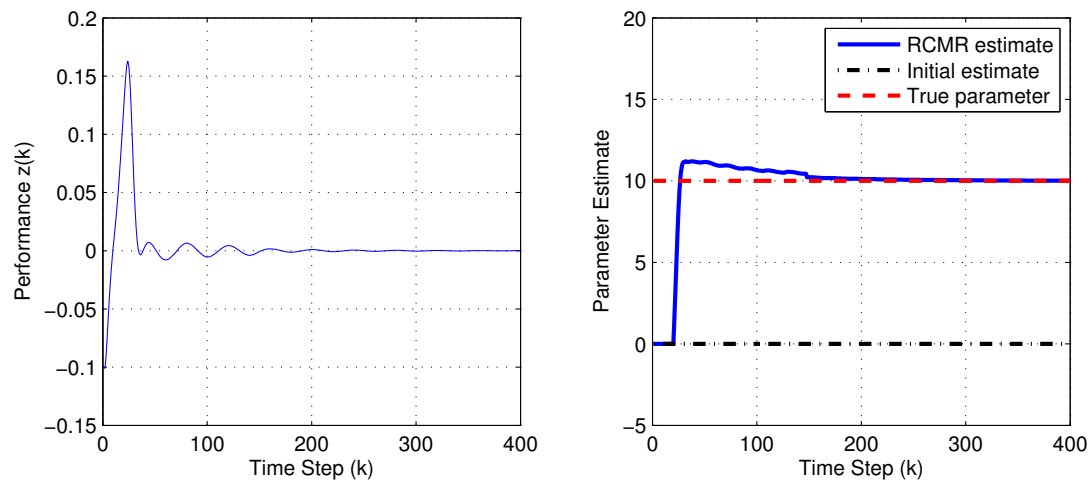

Figure 2. Example IV.1, performance $z(k)$ and estimated $\hat{k}$.

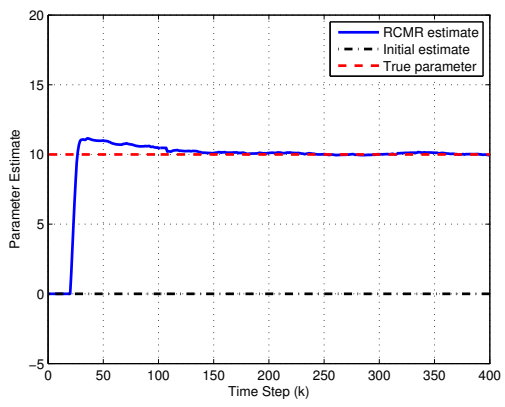

(a) $\mathrm{SNR}=100$

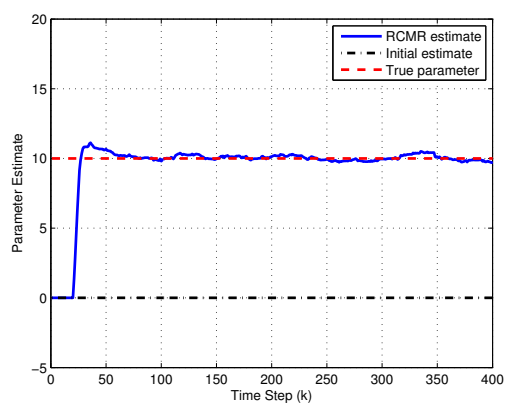

(b) $\mathrm{SNR}=10$

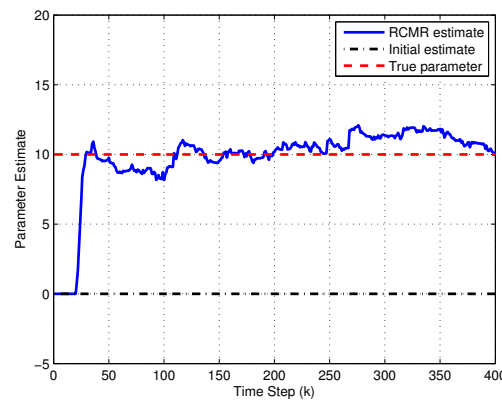

(c) $\mathrm{SNR}=1$

Figure 3. Example VI.1, the estimate $\hat{m}$ of $m$ for various values of SNR. As SNR decreases, the accuracy of the estimate is degraded.

where a parameter $\alpha$ in $A_{c}$ and $D_{c}$ is uncertain. Let $\alpha=\hat{\alpha}+\Delta \alpha$, where $\hat{\alpha}$ is the initial estimate of $\alpha$. The system model can be written approximately as

$$
\dot{x}=\left(A_{c}+\left.\frac{\partial A_{c}}{\partial \alpha}\right|_{\alpha=\hat{\alpha}} \Delta \alpha\right) x+\left(D_{c}+\left.\frac{\partial D_{c}}{\partial \alpha}\right|_{\alpha=\hat{\alpha}} \Delta \alpha\right) w .
$$

Discretizing (45) with sampling time $T_{s}$ yields

$$
\begin{aligned}
x(k+1) & =\left(I+A_{c} T_{s}+\left.\frac{\partial A_{c}}{\partial \alpha}\right|_{\alpha=\hat{\alpha}} \Delta \alpha T_{s}\right) x(k)+\left(D_{c} T_{s}+\left.\frac{\partial D_{c}}{\partial \alpha}\right|_{\alpha=\hat{\alpha}} \Delta \alpha T_{s}\right) w(k) \\
& =\left(A+\left.\frac{\partial A_{c}}{\partial \alpha}\right|_{\alpha=\hat{\alpha}} \Delta \alpha T_{s}\right) x(k)+\left(D_{1}+\left.\frac{\partial D_{c}}{\partial \alpha}\right|_{\alpha=\hat{\alpha}} \Delta \alpha T_{s}\right) w(k) .
\end{aligned}
$$

We use (46) as the true system model with uncertain parameter $\Delta \alpha$. Since our goal is to estimate $\Delta \alpha$, we rewrite $(46)$

$$
x(k+1)=A x+\left[\begin{array}{lll}
D_{1} & \left.\frac{\partial A_{c}}{\partial \alpha}\right|_{\alpha=\hat{\alpha}} T_{s} & \left.\frac{\partial D_{c}}{\partial \alpha}\right|_{\alpha=\hat{\alpha}} T_{s}
\end{array}\right]\left[\begin{array}{c}
w(k) \\
\Delta \alpha x(k) \\
\Delta \alpha w(k)
\end{array}\right] .
$$


Next, let

$$
\begin{aligned}
\hat{B} & =\left[\begin{array}{cc}
\left.\frac{\partial A_{c}}{\partial \alpha}\right|_{\alpha=\hat{\alpha}} T_{s} & \left.\frac{\partial D_{c}}{\partial \alpha}\right|_{\alpha=\hat{\alpha}} T_{s}
\end{array}\right], \\
\hat{y}(k) & =\left[\begin{array}{c}
\hat{x}(k) \\
w(k)
\end{array}\right], \\
\Delta \alpha(k) & =\hat{G}_{s}(q)=\theta(k), \\
\hat{u}(k) & =\Delta \alpha(k) \hat{y}(k) .
\end{aligned}
$$

Note that $(51)$ shows that $\hat{u}(k)$ is a function of $\hat{y}(k)$. We define

$$
\hat{Y}(k) \triangleq\left[\begin{array}{lll}
\hat{y}^{\mathrm{T}}\left(k-k_{1}\right) & \cdots & \hat{y}^{\mathrm{T}}\left(k-k_{s}\right)
\end{array}\right]^{\mathrm{T}} \in \mathbb{R}^{s l_{y}} .
$$

With these signals, (27)-(31) become

$$
\begin{aligned}
\bar{J}\left(\Delta \alpha^{*}(k-1), k\right) & =\mathcal{A}(k) \Delta \alpha^{* 2}(k-1)+\mathcal{B}(k) \Delta \alpha^{*}(k-1)+\mathcal{C}(k), \\
\mathcal{A}(k) & \triangleq \hat{Y}(k-1)^{T}\left[\tilde{\mathcal{H}}^{\mathrm{T}} R(k) \tilde{\mathcal{H}}+\eta(k) I_{l}\right] \hat{Y}(k-1), \\
\mathcal{B}(k) & \triangleq 2 \tilde{\mathcal{H}}^{\mathrm{T}} R(k)[Z(k)-\tilde{\mathcal{H}} \tilde{U}(k-1)] \hat{Y}(k-1), \\
\mathcal{C}(k) & \triangleq Z^{\mathrm{T}}(k) R(k) Z(k)-2 Z^{\mathrm{T}}(k) R(k) \tilde{\mathcal{H}} \tilde{U}(k-1)+\tilde{U}^{\mathrm{T}}(k-1) \tilde{\mathcal{H}}^{\mathrm{T}} R(k) \tilde{\mathcal{H}} \tilde{U}(k-1), \\
\tilde{U}^{*}(k-1) & =\Delta \alpha^{*}(k-1) \hat{Y}(k-1)=-\frac{1}{2} \mathcal{A}^{-1}(k) \mathcal{B}(k) \hat{Y}(k-1) .
\end{aligned}
$$

We use one parameter in $\tilde{U}^{*}(k-1)$ and the corresponding parameter in $\tilde{Y}(k-1)$ to execute the recursive least squares update. In the next section we use the same mass-spring system to illustrate the algorithm.

\section{Mass-Spring Example: Inertia Estimation}

Example VI.1. Consider the mass-spring-damper structure shown in Example IV.1 where $A_{c}=$ $\left[\begin{array}{cc}0 & 1 \\ -\frac{\kappa}{m} & -\frac{c}{m}\end{array}\right], D_{c}=\left[\begin{array}{c}0 \\ \frac{1}{m}\end{array}\right]$, and $E_{c}=\left[\begin{array}{ll}0 & 1\end{array}\right]$. In this example we assume that the parameter $m$ is unknown. We demonstrate the algorithm by choosing $\kappa=30$ and $c=5$, and we assume that $m=0.9$. We use an initial estimate is $\hat{m}(0)=1$, so that $\Delta m(0)=-0.1$.

From (48), we obtain

$$
\hat{B}=\left[\begin{array}{ccc}
0 & 0 & 0 \\
3 & 0.5 & -0.1
\end{array}\right]
$$

We choose $P(0)=1, \lambda=1, \eta=0, \mu=0, \sigma_{v}^{2}=0$ and $\tilde{\mathcal{H}}=\left[\begin{array}{ll}H_{1} & H_{2}\end{array}\right]$, which are the first and second Markov parameters of $\hat{G}$. We choose the ramp input $w(k)=0.1 k$ and the initial state $\left[\begin{array}{l}\hat{x}_{1}(0) \\ \hat{x}_{2}(0)\end{array}\right]=$ $\left[\begin{array}{l}x_{1}(0) \\ x_{2}(0)\end{array}\right]=\left[\begin{array}{l}0 \\ 0\end{array}\right]$. The system refinement algorithm is turned on at $k=100$ steps. Figure 4 shows the performance $z$ and the estimate $\hat{m}$.

We next consider the effect of sensor noise. We assume $\mu_{v}=0$. Figure 5 shows the estimation performance for several values of $\sigma_{v}^{2}$. 

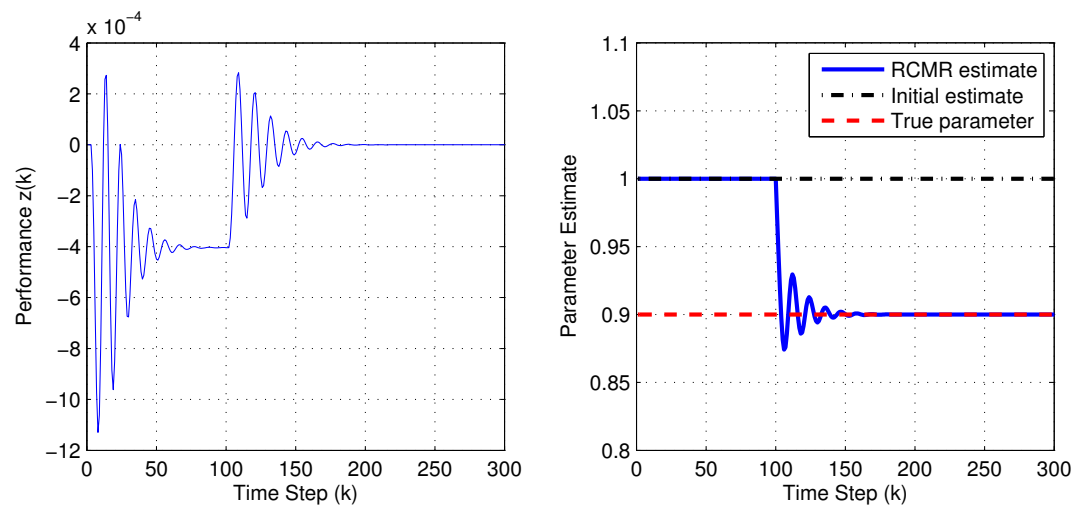

Figure 4. Example VI.1, the performance $z$ and the estimate $\hat{m}$.

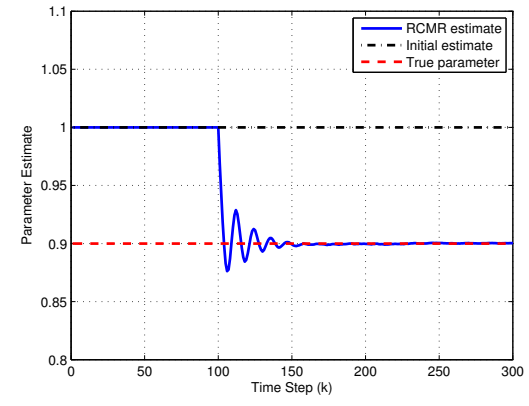

(a) $\sigma_{v}^{2}=1 \times 10^{-10}$

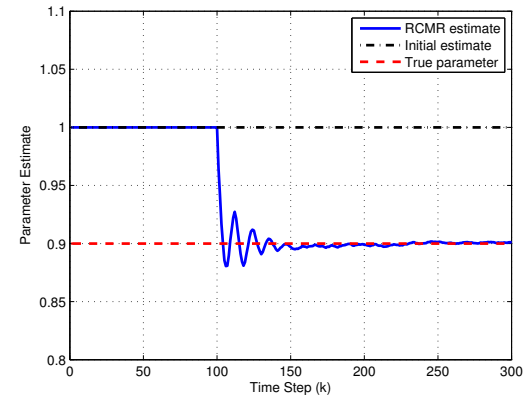

(b) $\sigma_{v}^{2}=1 \times 10^{-9}$

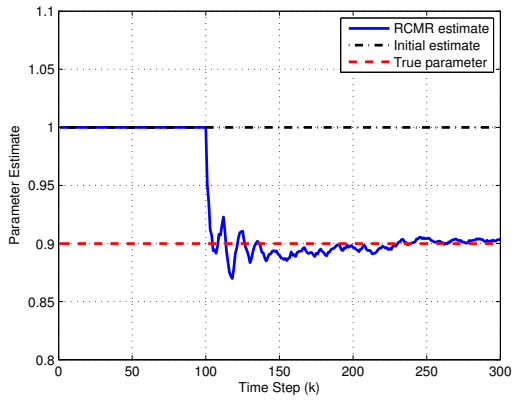

(c) $\sigma_{v}^{2}=1 \times 10^{-8}$

Figure 5. Example VI.1, the estimate $\hat{m}$ of $m$ for various values of $\sigma_{v}^{2}$. As $\sigma_{v}^{2}$ increases, the accuracy of the estimate is degraded.

\section{Aircraft Examples}

We now estimate stability parameters under various scenarios of measurements and measurement noise. Consider the linearized longitudinal B-767 model

$$
\left[\begin{array}{c}
\dot{u} \\
\dot{\alpha} \\
\dot{q} \\
\dot{\theta}
\end{array}\right]=\left[\begin{array}{cccc}
-0.0168 & 0.1121 & 0.0003 & -0.5608 \\
-0.0164 & -0.7771 & 0.9945 & 0.0015 \\
-0.0417 & -3.6595 & -0.9544 & 0 \\
0 & 0 & 1.0000 & 0
\end{array}\right]\left[\begin{array}{c}
u \\
\alpha \\
q \\
\theta
\end{array}\right]+\left[\begin{array}{cc}
-0.0243 & 0.0519 \\
-0.0634 & -0.0005 \\
-3.6942 & 0.0243 \\
0 & 0
\end{array}\right]\left[\begin{array}{c}
\delta e \\
\delta T
\end{array}\right],
$$

where $\delta e$ is the elevator deflection and $\delta T$ is the thrust perturbation. We discretize (59) using a zero-order hold with $T_{s}=0.1 s$, which yields

$$
A=\left[\begin{array}{cccc}
0.9983 & 0.0110 & -0.0022 & -0.0560 \\
-0.0018 & 0.9085 & 0.0907 & 0.0002 \\
-0.0037 & -0.3336 & 0.8924 & 0.0001 \\
-0.0002 & -0.0172 & 0.0948 & 1.0000
\end{array}\right], D_{1}=\left[\begin{array}{cc}
-0.0022 & 0.0052 \\
-0.0234 & 0.0001 \\
-0.3492 & 0.0023 \\
-0.0178 & 0.0001
\end{array}\right],
$$

and $w=\left[\begin{array}{cc}\delta e & \delta T\end{array}\right]^{\mathrm{T}}$ is the system input. For all examples in this section, RCMR is turned on at $k=100$ steps, and $\hat{x}(0)=x(0)=\left[\begin{array}{cccc}0 & 0 & 0 & 0\end{array}\right]^{\mathrm{T}}$. We choose $\delta e$ and $\delta T$ to be zero-mean white Gaussian noises with variance 0.001 . 
Example VII.1. Assume an initial guess of $A(3,3)=0.8000$. The true value of $A(3,3)$ is 0.8924 and $\Delta A(3,3)=0.0924$. Our goal is to identify $A(3,3)$. We choose $E_{1}=\left[\begin{array}{llll}0 & 0 & 1 & 0\end{array}\right]$ so that $\bar{y}_{0}=q$ and $\hat{y}_{0}=\hat{q}$. In this example, $\hat{A}=A, \hat{D}_{1}=D_{1}, \hat{E}_{1}=E_{1}, \hat{B}=\left[\begin{array}{llll}0 & 0 & 1 & 0\end{array}\right]^{\mathrm{T}}$ and $\hat{C}=\left[\begin{array}{cccc}0 & 0 & 1 & 0\end{array}\right]$. In this model, $n_{c}=0$ and $\Delta \hat{A}(3,3)=\theta(k)$. We choose $P(0)=200, \lambda=0.97, \eta=0, \mu_{v}=0, \sigma_{v}^{2}=0$ and $\tilde{\mathcal{H}}=\left[\begin{array}{ll}H_{1} & H_{2}\end{array}\right]$, which are the first and second Markov parameters of $\hat{G}$. The parameters of the model refinement algorithm are chosen such that $z(k)$ is minimized. Figure 6 shows the performance $z$ and the estimated $\hat{A}(3,3)$.
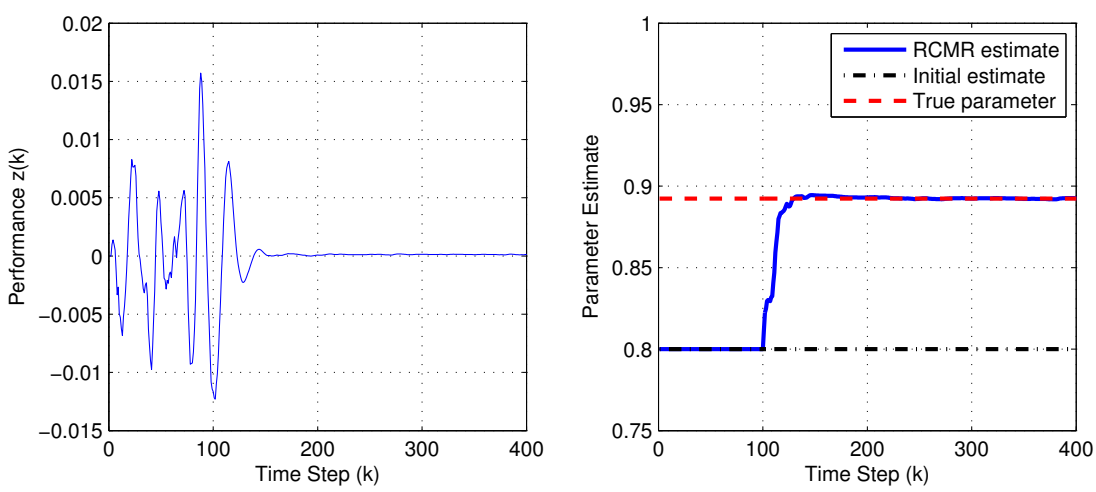

Figure 6. Example VII.1, performance $z(k)$ and estimated $\hat{A}(3,3)$.

Example VII.2. We next consider the case where multiple parameters in $A$ are possibly uncertain and $K=\Delta A$ has the form shown. We assume full state feedback and $y=\left[\begin{array}{llll}u & \alpha & q & \theta\end{array}\right]^{\mathrm{T}}$. Thus $\hat{A}_{\text {closed-loop }}=\hat{A}+\hat{B} \hat{K} \hat{C}$, where $\hat{B}=\hat{C}=I_{4}$ and $\hat{K}(k)=\theta(k)$, where

$$
K=\left[\begin{array}{cccc}
-0.05 & -0.002 & 0 & 0 \\
0 & -0.04 & 0 & 0 \\
0 & -0.04 & -0.04 & 0 \\
0 & 0 & 0 & -0.04
\end{array}\right] \text {. }
$$

We choose $P(0)=100 I_{4}, \lambda=0.98, \eta=1, \mu_{v}=0, \sigma_{v}^{2}=0$ and $\tilde{\mathcal{H}}=\left[\begin{array}{cc}H_{1} & H_{2}\end{array}\right]$. Figure 7 shows the performance $z(k)$ and the parameter estimates $\theta(k)$. The parameter error $\|\theta(k)-K\|_{2}$ converges to zero.
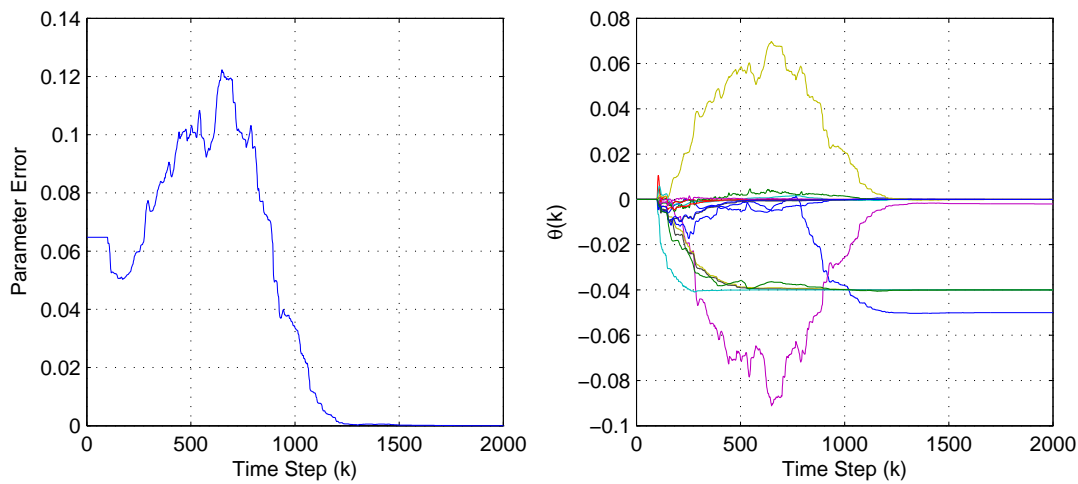

Figure 7. Example VII.2, parameter error $\|\theta(k)-K\|_{2}$ and estimated $\theta(k)$.

Example VII.3. We next consider the case where parameters of the $A$ matrix that are not being estimated have modeling errors. We assume $\hat{A}(3,2)=-0.3000$, whereas the true value is -0.3336 . We 
assume that this modeling error is unknown and that our goal is to identify $A(3,3)$. We choose $P(0)=100$, $\lambda=1, \eta=0, \mu_{v}=0, \sigma_{v}^{2}=0$ and $\tilde{\mathcal{H}}=\left[\begin{array}{ll}H_{1} & H_{2}\end{array}\right]$, which are the first and second Markov parameters of $\hat{G}$. Figure 8 shows that the modelling errors degrade the accuracy of the estimates of the targeted parameters.
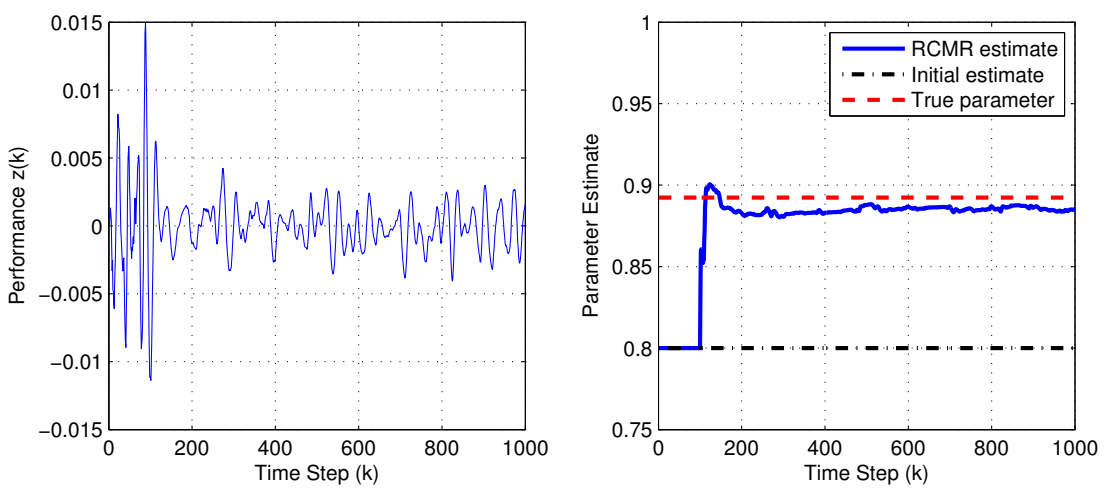

Figure 8. Example VII.3, performance $z$ and estimated $\hat{A}(3,3)$.

Example VII.4. We next consider the case where the unknown parameter is time-varying. In this example, we assume $A(3,3)$ is time-varying, $\hat{A}=A, \hat{D}_{1}=D_{1}, \hat{E}_{1}=E_{1}, \hat{B}=\left[\begin{array}{cccc}0 & 0 & 1 & 0\end{array}\right]^{\mathrm{T}}$, and $\hat{C}=\left[\begin{array}{llll}0 & 0 & 1 & 0\end{array}\right]$. Figure 9 shows the performance when $A(3,3)$ is varying at a constant rate, and Figure 10 shows the performance when $A(3,3)$ is varying as a sinusoidal signal. In both cases, $P(0)=10$, $\lambda=0.98, \eta=0, \mu_{v}=0, \sigma_{v}^{2}=0$ and $\tilde{\mathcal{H}}=\left[\begin{array}{ll}H_{1} & H_{2}\end{array}\right]$.
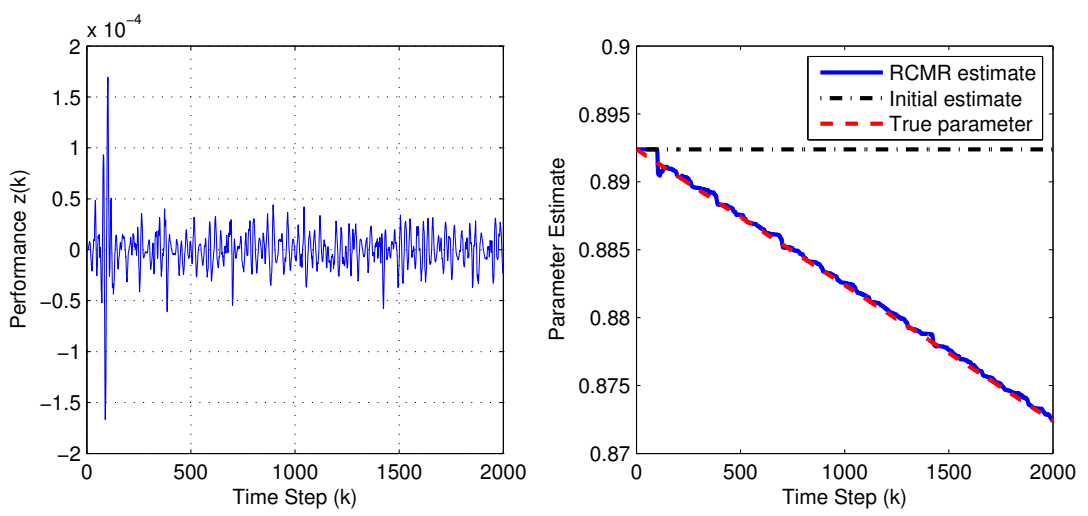

Figure 9. Example VII.4, performance $z$ and estimated $\hat{A}(3,3) . A(3,3)$ is the ramp signal $A(3,3)=0.8924-10^{-5} k$, $P(0)=10, \lambda=0.98, \eta=0, \mu_{v}=0, \sigma_{v}^{2}=0$ and $\tilde{\mathcal{H}}=\left[H_{1}, H_{2}\right]$.

Example VII.5. We next consider Example VII.1 with sensor noise present. We assume $\mu_{v}=0$. Figure 11 shows the estimation performance for several values of SNR. As the SNR decreases, the accuracy of the parameter estimatition degrades and convergence time increases.

Example VII.6. We next consider Example VII.1 with non-zero-mean measurement noise. We assume $\mathrm{SNR}=100$ and compare the estimate for several values of $\mu_{v}$. Figure 12 shows the affect on estimation performance as $\mu_{v}$ increases. As the bias $\mu_{v}$ increases, the accuracy of the parameter estimation degrades and convergence time increases. 

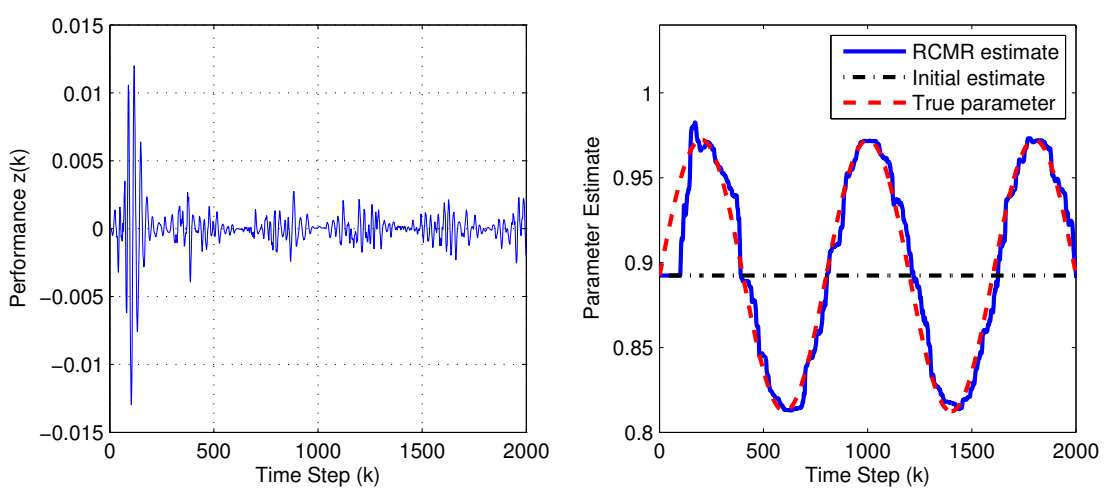

Figure 10. Example VII.4, performance $z$ and estimated $\hat{A}(3,3) . \quad A_{d}(3,3)$ is the sinusoidal signal $A(3,3)=$ $0.8924+0.08 \sin (\pi k / 400), P(0)=10, \lambda=0.98, \eta=0, \mu_{v}=0, \sigma_{v}^{2}=0$ and $\tilde{\mathcal{H}}=\left[H_{1}, H_{2}\right]$.

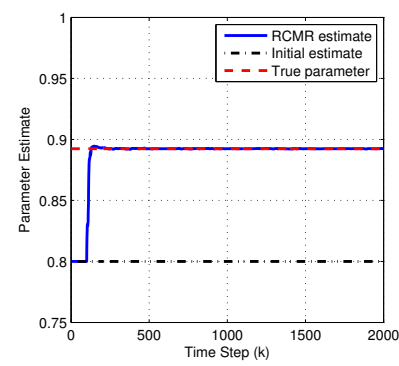

(a) $\mathrm{SNR}=\infty$

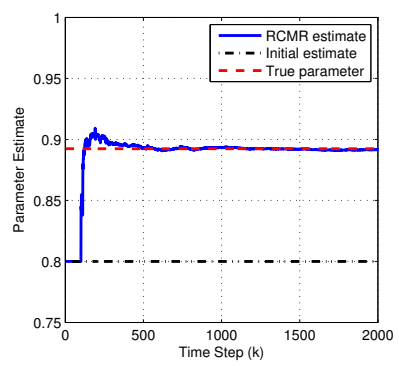

(b) $\mathrm{SNR}=100$

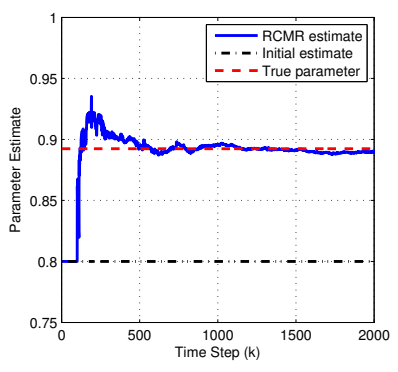

(c) $\mathrm{SNR}=10$

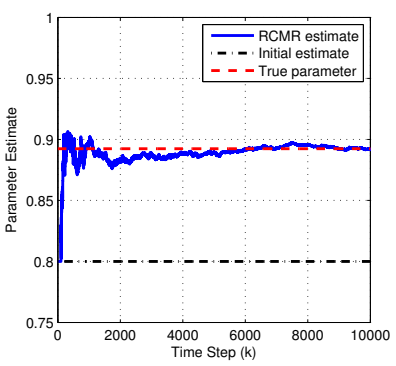

(d) $\mathrm{SNR}=1$

Figure 11. Example.VII.5. (a) $P(0)=200, \lambda=0.97$. (b) $P(0)=200, \lambda=1$. (c) $P(0)=100, \lambda=1$. (d) $P(0)=15$, $\lambda=1$. In all cases, $\eta=0$, and $\tilde{\mathcal{H}}=\left[H_{1}, H_{2}\right]$.

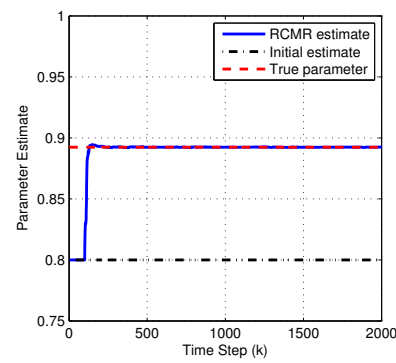

(a) $\mu_{v}=0$

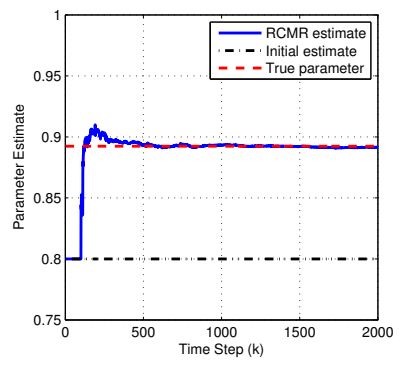

(b) $\mu_{v}=-0.001$

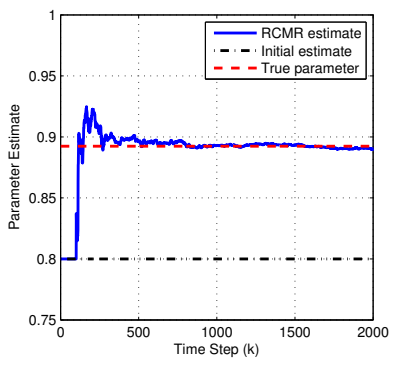

(c) $\mu_{v}=-0.01$

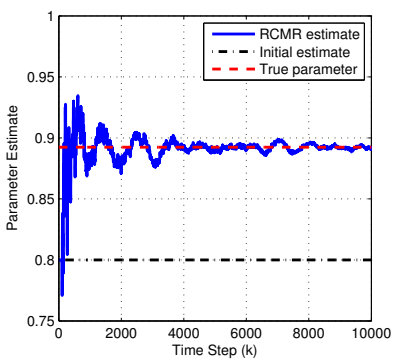

(d) $\mu_{v}=-0.1$

Figure 12. Example.VII.6. (a) $P(0)=200, \lambda=0.97$. (b) $P(0)=200, \lambda=1$. (c) $P(0)=200, \lambda=1$. (d) $P(0)=20$, $\lambda=1$. In all cases, $\eta=0$, and $\tilde{\mathcal{H}}=\left[H_{1}, H_{2}\right]$.

\section{Airspeed Estimation}

In this section we use RCMR to estimate airspeed.

Example VIII.1. Consider the linearized longitudinal transfer functions [16] for a typical business 
jet in cruise given by

$$
\left[\begin{array}{c}
\dot{u} \\
\dot{\alpha} \\
\dot{q} \\
\dot{\theta}
\end{array}\right]=\left[\begin{array}{cccc}
X_{u_{0}}+X_{T_{u_{0}}} & X_{\alpha_{0}} & X_{q_{0}} & -g \\
\frac{Z_{u_{0}}+Z_{T_{u_{0}}}}{U_{0}} & \frac{Z_{\alpha_{0}}}{U_{0}} & \frac{U_{0}+Z_{q_{0}}}{U_{0}} & 0 \\
M_{u_{0}}+M_{T_{u_{0}}} & M_{\alpha_{0}}+M_{T_{\alpha_{0}}} & M_{q_{0}} & 0 \\
0 & 0 & 1 & 0
\end{array}\right]\left[\begin{array}{c}
u \\
\alpha \\
q \\
\theta
\end{array}\right]+\left[\begin{array}{c}
X_{\delta e_{0}} \\
\frac{Z_{\delta e_{0}}}{U_{0}} \\
M_{\delta e_{0}} \\
0
\end{array}\right] \delta e,
$$

where $U_{0}$ is the aircraft speed at which the equations of motion of the aircraft are linearized. $X, Z, M$ are the related coefficients of the aircraft dynamic mode and many of these coefficients are depend on $U_{0}$. Using the relations of these coefficients to $U_{0},(62)$ can be written as

$$
\left[\begin{array}{c}
\dot{u} \\
\dot{\alpha} \\
\dot{q} \\
\dot{\theta}
\end{array}\right]=\left[\begin{array}{cccc}
-\frac{4.9950}{U_{0}} & 8.9782 & 0 & -32.1522 \\
-\frac{93.8250}{U_{0}^{2}} & -\frac{445.7224}{U_{0}} & 1-\frac{1255.3650}{U_{0}^{2}} & 0 \\
\frac{0.6075}{U_{0}} & -7.4416 & -\frac{634.2975}{U_{0}} & 0 \\
0 & 0 & 1 & 0
\end{array}\right]\left[\begin{array}{c}
u \\
\alpha \\
q \\
\theta
\end{array}\right]+\left[\begin{array}{c}
0 \\
-\frac{42.1968}{U_{0}} \\
-17.6737 \\
0
\end{array}\right] \delta e
$$

The goal is to estimate $U_{0}$. The true value of $U_{0}(0)$ is $625 \mathrm{ft} / \mathrm{s}$, and we use the initial estimate $\hat{U}_{0}=675$ $\mathrm{ft} / \mathrm{s}$. We choose various $\bar{y}_{0}$, and compare the performance of the algorithm.

Figure 13 shows the performance of RCMR with $\bar{y}_{0}=\alpha$. In this case, the input $w(k)=0.0008$, $P(0)=0.0003, \lambda=0.9999, \eta=0, \mu=0, \sigma_{v}^{2}=0$ and $\tilde{\mathcal{H}}=\left[\begin{array}{lll}H_{1} & \cdots & H_{12}\end{array}\right]$. Figure 14 shows the performance of RCMR with $\bar{y}_{0}=q$. In this case, the input $w(k)=10^{-7} k, P(0)=1, \lambda=0.99, \eta=0, \mu=0$, $\sigma_{v}^{2}=0$ and $\tilde{\mathcal{H}}=\left[\begin{array}{ll}H_{1} & H_{2}\end{array}\right]$. Figure 15 shows the performance of RCMR with $\bar{y}_{0}=\theta$. In this case, the input $w(k)=10^{-7} k, P(0)=0.01, \lambda=0.999, \eta=0, \mu=0, \sigma_{v}^{2}=0$, and $\tilde{\mathcal{H}}=\left[\begin{array}{ll}H_{1} & H_{2}\end{array}\right]$.
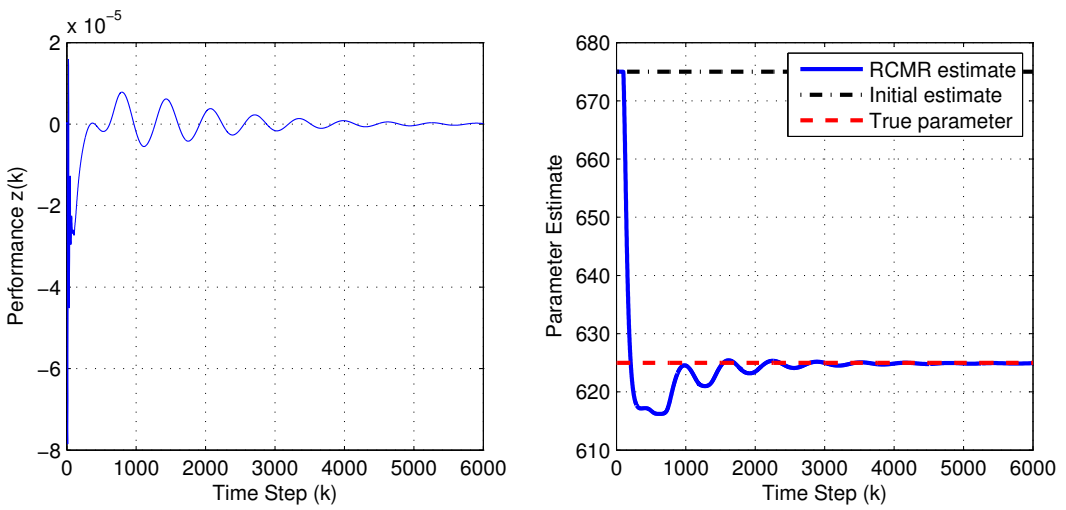

Figure 13. Airspeed estimation with $\bar{y}_{0}=\alpha$. The performance $z$ and the airspeed estimate $\hat{U}_{0}$ are shown.

\section{Conclusions}

This paper showed that RCMR can be used to estimate unknown parameters in a state space model. Both constant and time-varying parameters were considered, under various sensor noise levels and choices of measurements. Future work will compare the accuracy of this technique to nonlinear estimation methods.

\section{Acknowledgment}

This work was supported in part by the National Aeronautics and Space Administration under Cooperative Agreement NNX12AM54A. 

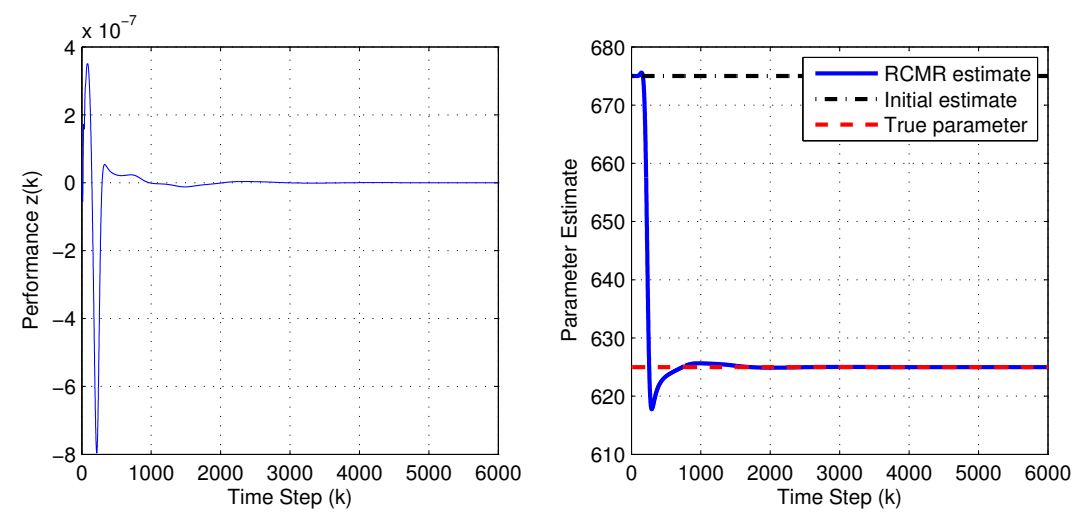

Figure 14. Airspeed estimation with $\bar{y}_{0}=q$. The performance $z$ and the airspeed estimate $\hat{U}_{0}$ are shown.
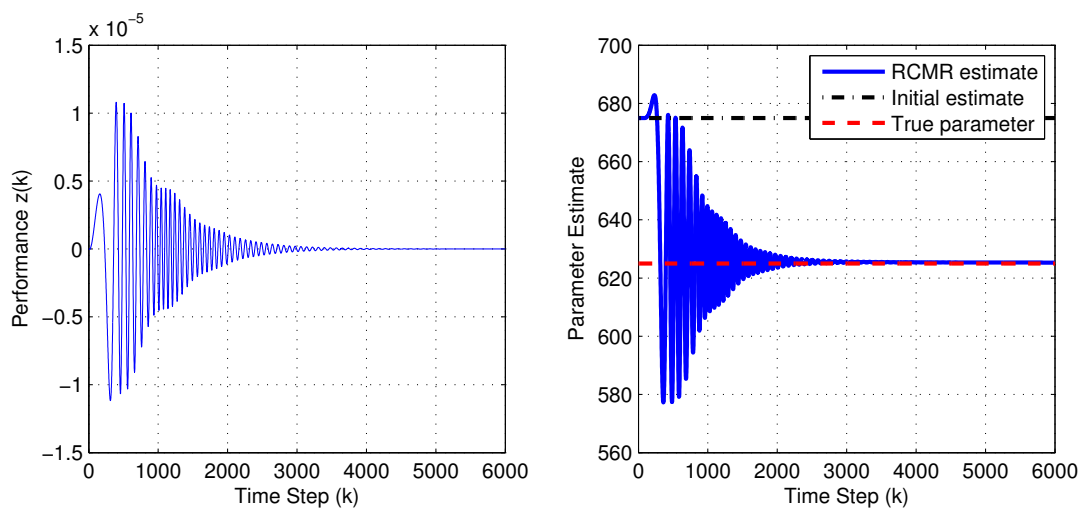

Figure 15. Airspeed estimation with $\bar{y}_{0}=\theta$. The performance $z$ and the airspeed estimate $\hat{U}_{0}$ are shown.

\section{References}

${ }^{1}$ Palanthandalam-Madapusi, H., Renk, E. L., and Bernstein, D. S., "Data-Based Model Refinement for Linear and Hammerstein Systems Using Subspace Identification and Adaptive Disturbance Rejection," Proc. Conf. Contr. Appl., Toronto, Canada, August 2005, pp. 1630-1635.

${ }^{2}$ D'Amato, A. M., Arritt, B. J., Banik, J. A., Ardelean, E. V., and Bernstein, D. S., "Structural Health Determination and Model Refinement for a Deployable Composite Boom," Proc. AIAA SDM Conf., Palm Springs, CA, April 2009, AIAA2009-2373.

${ }^{3}$ Aljanaideh, K., Ali, A. A., Holzel, M., Kukreja, S., and Bernstein, D. S., "Sensor-to-Sensor Identification of Hammerstein Systems," Proc. Conf. Dec. Contr., Maui, HI, December 2012.

${ }^{4}$ Haroon, Y. W. L. M., Adams, D. E., and Ferri, A. A., "A Time and Frequency Domain Approach For Identifying Nonlinear Mechanical System Models in the Absence of an Input Measurement," Sound and Vibration, Vol. 283, 2005, pp. 11371155.

${ }^{5}$ Moheimani, S. O. R., "Model Correction for Sampled-data Models of Structures," Journal of Guidance, Control, and Dynamics, Vol. 24, 2001, pp. 634-637.

${ }^{6}$ Danforth, C. M., Kalnay, E., and Miyoshi, T., "Estimating and Correcting Global Weather Model Error," Monthly Weather Rev., Vol. 135, 2007, pp. 281-299.

${ }^{7}$ Ljung, L., "Asymptotic Behavior of the Extended Kalman Filter as a Parameter Estimator for Linear Systems," IEEE Trans. Autom. Control, Vol. 24, 1979, pp. 36-50.

${ }^{8}$ Ristic, B., Arulampalam, S., and Gordon, N., Beyond the Kalman Filter, Artech House, 2004.

${ }^{9}$ Julier, S., Uhlmann, J., and Durrant-Whyte, H., "A New Method for the Nonlinear Transformation of Means and Covariances in Filters and Estimators," IEEE Trans. Autom. Control, Vol. 45, 2000, pp. 477-482.

${ }^{10}$ Psiaki, M., "Backward-Smoothing Extended Kalman Filter," AIAA J. Guid. Contr. Dyn., Vol. 28, 2005, pp. 885-894.

${ }^{11}$ Rao, C., Rawlings, J., and Mayne, D., "Constrained State Estimation for Nonlinear Discrete-Time Systems: Stability and Moving Horizon Approximations," IEEE Trans. Autom. Control, Vol. 48, 2003, pp. 246-258.

${ }^{12}$ Santillo, M. A., D'Amato, A. M., and Bernstein, D. S., "System Identification Using a Retrospective Correction Filter for Adaptive Feedback Model Updating," Proc. Amer. Contr. Conf., St. Louis, MO, June 2009, pp. $4392-4397$. 
${ }^{13}$ D'Amato, A. M. and Bernstein, D. S., "Linear Fractional Transformation Identification Using Retrospective Cost Optimization," Proc. SYSID, Saint-Malo, France, July 2009, pp. 450-455.

${ }^{14}$ D'Amato, A. M., Wu, A. R., Mitchell, K. S., Kukreja, S. L., and Bernstein, D. S., "Damage Localization for Structural Health Monitoring Using Retrospective Cost Model Refinement," Proc. AIAA SDM Conf., Orlando, FL, April 2010, AIAA2010-2628.

${ }^{15}$ D'Amato, A. M., Ridley, A. J., and Bernstein, D. S., "Retrospective-Cost-Based Adaptive Model Refinement for the Ionosphere and Thermosphere," Statistical Analysis and Data Mining, Vol. 4, 2011, pp. 446-458.

${ }^{16}$ Roskam, J., Airplane Flight Dynamics and Automatic Flight Control, DARcorp, 2001. 